\title{
Die Utopie einer günstigeren Gesundheitsversorgung
}

\author{
Hans Hoppelera, Jürg Müller ${ }^{b}$, Marc Lauper $^{c}$ \\ a Prof. Dr. med. emerit., Bolligen; ${ }^{b}$ Dr., Kirchdorf; ${ }^{c}$ Stettlen
}

\section{Das Sparpotential}

Ohne Einbusse an Qualität könnten in der Schweiz $10 \%$ der Kosten des Gesundheitswesens, also zwischen 6 und 7 Milliarden Franken, eingespart werden. Dies hält der von der Schweizerischen Akademie der Wissenschaften 2012 publizierte Bericht zu «Effizienz, Nutzung und Finanzierung des Gesundheitswesens» fest [1]. Man wundert sich, dass trotz eines enormen Sparpotentials die Gesundheitskosten und damit die Krankenkassenprämien und die über Steuern finanzierten Beiträge der Kantone an die Spitäler jährlich weiter steigen. Der Wunsch oder der Druck, zu sparen, scheint demnach nicht besonders gross. Ein Grund mag darin liegen, dass die Schweizer gesamthaft gesehen mit dem teuersten Gesundheitswesen Europas [2] zufrieden sind. Der finanzielle Aufwand scheint sich nicht zuletzt deswegen zu lohnen, weil sich die Schweizer damit die (beinahe) höchste Lebenserwartung bei Geburt und die höchste mit 65 Jahren erkaufen.

Der Bericht der Schweizerischen Akademie der Wissenschaften geht davon aus, dass das Sparpotential von 6-7 Milliarden Franken ohne Verlust an Qualität erreichbar wäre. Das heisst, die hohe Zufriedenheit der Schweizer mit dem Gesundheitswesen und unsere hohe Lebenserwartung wären deutlich günstiger zu haben. Warum werden also mögliche Einsparmöglichkeiten nicht wahrgenommen? Dies mag vor allem daran liegen, dass die wesentlichen Akteure im Ge-

\section{L'utopie de soins de santé moins chers}

Chaque année, nous constatons une nouvelle hausse des primes des caisses d'assurancemaladie. En Suisse, le secteur de la santé relève de la responsabilité des cantons. Ces derniers devraient certes, en vertu des dispositions légales, veiller à ce que les soins de santé soient avantageux en termes de coût. Mais dans les faits, cela leur est impossible. En tant que propriétaires des hôpitaux, ils sont en effet également responsables de la régulation et du contrôle de leurs propres hôpitaux ainsi que des établissements privés et assument un rôle de payeurs. La concurrence dans les soins hospitaliers souhaitée par l'Assemblée fédérale est une illusion, car notre système de santé social présente toutes les caractéristiques principales d'une économie planifiée, comme des prix fixes, définis dans les DRG. II ne faut pas s'attendre à un quelconque changement dans la hausse constante des coûts de santé, car elle profite aux médecins, aux hôpitaux et aux caisses-maladie. De leur côté, les cantons ne prennent guère de mesures efficaces et les patients et contribuables n'ont pas de lobby.

sundheitswesen keinen Grund zum Sparen sehen. Letztlich profitieren alle, die Ärzte, die Krankenkassen und die Spitäler, von immer höheren Gesundheitskosten. Interessanterweise hat der Patient, der über die Krankenkassenprämien und die Steuern das Gesundheitswesen finanziert, dazu nichts zu sagen. Es gibt keine Lobby für den Patienten und Steuerzahler. Patienten sind in den relevanten Gremien des Bundes und den Gremien der für das Gesundheitswesen direkt verantwortlichen Kantone nicht vertreten. Die Schweizerischen Patientenorganisationen beschäftigen sich hauptsächlich mit Fragen der Patientenrechte - im Zusammenhang mit der Finanzierung unseres Gesundheitswesens ein Aspekt von untergeordneter Bedeutung.

Gesundheit ist in der Schweiz eine kantonale Angelegenheit. Es wäre deswegen an den verantwortlichen kantonalen Organen, dafür zu sorgen, dass die Gesundheitskosten nicht ungebremst steigen. Die kantonalen Gesetze verlangen, dass Gesundheitsleistungen kostengünstig zu erbringen sind. So hält zum Beispiel das Spitalversorgungsgesetz des Kantons Bern im Artikel 3 Absatz 1 fest: "Die Spitalversorgung und das Rettungswesen sind allgemein zugänglich, bedarfsgerecht, von guter Qualität und wirtschaftlich.» Der Kanton verpflichtet sich überdies im selben Artikel, dass die eingesetzten Mittel optimal wirken. Angesichts des offensichtlich enormen Sparpotentials im Gesundheitswesen scheint dem Thema «Wirtschaftlichkeit des Gesundheitswesens» auf kantonaler Ebene allerdings nicht eine hohe Priorität eingeräumt zu werden.

Die kantonale Untätigkeit hat Gründe; die Kantone sind in einer ausserordentlich schwierigen Situation. Es sind die kantonalen Gesundheitsdirektionen, welche das Gesundheitswesen und speziell die Spitäler regulieren und kontrollieren. Dabei betrifft die Regulation und Kontrolle nicht nur die öffentlichen Spitäler, sondern auch deren direkte Konkurrenten, die Privatspitäler. Zusätzlich finanzieren die Kantone zu 55\% über DRG gleichermassen die öffentlichen wie die Privatspitäler. Dieser Umstand wird weiter dadurch verschlimmert, dass die Kantone auch Besitzer der öffentlichen 
Spitäler sind. Also jedes Interesse haben müssen, dass diese Spitäler finanziell erfolgreich operieren. Diese Alptraumsituation einer verantwortungsbewussten Regierungsführung (governance) und die damit verbundenen Zielkonflikte lassen ein wirkungsvolles Eingreifen der Kantone gegen die laufende Zunahme der Gesundheitskosten offensichtlich aktuell nicht zu.

\section{Ein utopischer Aktionsplan}

In der Wirtschaft wäre es aus Konkurrenzgründen fahrlässig, ein Sparpotential von 10\% nicht zu realisieren. In der Politik ist dies zulässig, da bei steigenden Kosten sowohl Steuern als auch Krankenkassenprämien nach oben angepasst werden können. Trotz dieser, für unsere Gesundheitswirtschaft günstigen, Sachlage kann man sich fragen, was getan werden könnte, um das identifizierte Sparpotential zu realisieren. Die Schweizerische Akademie der Wissenschaften hat in ihrem Bericht [1] das Einsparpotential den Bereichen Überversorgung, Unterversorgung, verzerrte Preise und Ineffizienz zugeordnet. Man kann einen Schritt weiter gehen und sich fragen, wer von welchem Ein-

\section{Eine Schwachstellenanalyse des Schweizer Gesundheitswesens zeigt, dass das grösste Einsparpotential bei den Kantonen liegt.}

sparpotential betroffen ist, respektive wer etwas tun könnte, um erkannte Schwachstellen im Gesundheitswesen zu eliminieren. Unter den verantwortlichen Akteuren treten die Kantone zweimal auf, einmal strategisch als verantwortliche Regulatoren und einmal operationell als Besitzer und Betreiber von Spitälern. Weitere Verursacher sind die Spitäler, die Ärzte, die Industrie resp. die Apotheken sowie die Patienten. Jede identifizierte Schwachstelle oder Lücke müsste mit einer geeigneten Aktion behoben werden. Eine Schwachstellenanalyse des Schweizer Gesundheitswesens zeigt, dass das grösste Einsparpotential bei den Kantonen liegt, welche es durch sinnvollere Regulation in der Hand hätten, Kosteneinsparungen zu bewirken. Die übrigen Verursacher tragen etwas weniger zu den kostspieligen Lücken in unserem Gesundheitswesen bei. Die Erfahrung zeigt, dass Aktionen zur Elimination von Schwachstellen nur dann erfolgreich verlaufen, wenn die Schwachstellen von allen Verursachern erkannt sind und diese bei der Behebung frühzeitig miteinbezogen werden. Dies verbietet Top-down-Aktionen im Gesundheitswesen. Die Erfahrungen aus der Industrie zeigen aber auch, dass bestenfalls 50\% eines identifizierbaren Einsparpotentials effektiv realisiert werden können. Immer noch ein beachtlicher Betrag.

\section{Die Rolle der Kantone}

Es wäre an den für das Gesundheitswesen verantwortlichen Kantonen, ein finanzielles Sanierungsprogramm an die Hand zu nehmen und aus den Dutzenden von möglichen Aktionen eine Prioritätenliste zu erstellen. Kriterien für eine erfolgreich anzugehende Schwachstellenelimination wären einerseits das Einsparpotential und anderseits die politische Machbarkeit. Zudem müssten die notwendigen Ressourcen (personelle und finanzielle) bereitgestellt werden und Verantwortlichkeiten für Einsparprojekte benannt werden.

Eine unabdingbare Voraussetzung für ein gezieltes Vorgehen der Kantone wäre das Vorhandensein einer Vision des Gesundheitswesens. Damit würden alle Akteure den politischen Willen in der Gestaltung des Gesundheitswesens kennen. Es müsste eine aus der Vision abgeleitete Strategie vorhanden sein, welche es gestatten würde, in allen Bereichen und für alle Akteure operationelle Ziele zu definieren. Diese Hausaufgaben haben nicht alle Kantone in gleichem Umfang gemacht. Die aktuelle Gesundheits- und Spitalpolitik vieler Kantone ist charakterisiert durch pragmatische Einzelaktionen. Gesundheitspolitische Einzelentscheide werden häufig nach aktueller Dringlichkeit und ohne Überprüfung der Auswirkungen auf das Gesamtsystem gefällt [3, 4]. Als Konsequenz ergeben sich daraus kantonale Unterschiede in den obligatorischen Gesundheitsausgaben für Haushalte von mehreren tausend Franken zwischen den günstigsten und den teuersten Kantonen [5].

Es gibt eine Reihe von systeminhärenten Problemen, welche von allen Kantonen angegangen werden müssten, wäre denn ein Abflachen des Anstiegs der Gesundheitskosten tatsächlicher politischer Wille.

Corporate Governance: Wie oben angesprochen, sind die Kantone die Besitzer der öffentlichen Spitäler. Gleichzeitig bestimmen sie mit Gesetzen und Verordnungen die strategischen Randbedingungen, unter denen die öffentlichen sowie die privaten Spitäler operieren, und finanzieren diese zudem zu 55\% über Steuergelder. Die Kantone vermischen damit die Belange der Strategie, Operation, Regulierung und der Kontrolle [5]. Grundsätzlich gibt es in dieser Situation zwei Lösungsmöglichkeiten:

- Die Kantone verzichten auf die Regulierung und behalten den Besitz der öffentlichen Spitäler.

- Die Kantone behalten die Regulierungshoheit und privatisieren die öffentlichen Spitäler.

Bei einer Abgabe der Regulierung müsste Letztere wohl vom BAG wahrgenommen werden. Dies würde das Ende der föderalistischen Regelung des schweize- 
rischen Gesundheitswesens bedeuten und scheint damit politisch nicht machbar. Eine Privatisierung der öffentlichen Spitäler, allenfalls mit Ausnahme der Universitätsspitäler, erscheint eher möglich. Dieses Vorgehen würde unter anderem auch der Corporate Governance [6] genügen. Die Kantone wären gezwungen, 51\% der Aktien der öffentlichen Spitäler zu veräussern, bekämen damit aber die Freiheit, ihre Strategie und eine damit verbundene Leistungsallokation bei allen Spitälern gleichermassen durchzusetzen.

Freie Marktwirtschaft vs. Planwirtschaft: Ein soziales Gesundheitswesen kann den Gesetzen des freien Markts nicht gehorchen. Das beginnt damit, dass sich auch die zahlungsschwächsten Glieder der Gesellschaft die teuersten Krankheiten «leisten». Als Konsequenz weist das Gesundheitswesen in der Schweiz die wesentlichen Merkmale einer Planwirtschaft auf. Die Kantone bestimmen mit einem Leistungsvertrag die Leistungen, welche die öffentlichen Spitäler zu erbringen haben. Die Preise sind dabei durch DRG (also Fallpauschalen) festgesetzt. Dies allein hebelt jegliche Marktfunktionen aus. Mit DRG werden auch im Umfang von 10\% der Fallpauschale die Investitionen abgegolten. Wir stellen fest, dass dieses System mannigfachst umgangen wird. Die Kantone (und Einzelspitäler) haben seit Einführung von DRG zusätzliche private und öffentliche Investitionsmittel in Milliardenhöhe und auf unterschiedlichstem Weg in ihre Institutionen fliessen lassen. Sie haben damit den vom Parlament angestrebten «Wettbewerb» verzerrt. Im ambulanten Bereich gibt es noch keine Fallpauschalen, sondern Einzelleistungen werden durch einen ebenfalls festgesetzten Tarif, den TARMED, abgegolten.

\section{Es ist nicht zu übersehen, dass der "Wett- bewerb» unter den Spitälern vor allem darin besteht, die Fallzahlen zu erhöhen.}

Dabei werden TARMED-Leistungen vollständig von den Krankenkassen getragen, während sich der Kanton am DRG mit 55\% beteiligt. Diese unterschiedliche Finanzierungsart ist ein weiterer unnötiger Fehlanreiz in unserem Gesundheitswesen [7]. Es ist nicht zu übersehen, dass der «Wettbewerb» unter den Spitälern vor allem darin besteht, die Fallzahlen zu erhöhen - bei gleichzeitiger Reduktion der Spitalaufenthaltsdauer und kreativer Rechnungsstellung. Im ambulanten Bereich sind ebenfalls deutliche Anzeichen einer Mengenausweitung besonders bei den Spezialärzten zu sehen. Dies zum Teil als Folge der bundesrätlich verordneten Besserstellung der Hausärzte. Da unser Gesundheitswesen alle Charakteristika einer Planwirtschaft trägt, wäre es angebracht, dies anzuerkennen und diese Planwirtschaft wenigstens intelligent zu betreiben.

Management-Informationssystem (MIS): Eine unabdingbare Voraussetzung für eine Verbesserung des Gesundheitswesens ist eine Rechnungslegung auf Stufe Kanton und Spital, welche es gestattet, geplante Vorhaben wirtschaftlich unter unterschiedlichen Szenarien durchzurechnen, um eine zukünftige Rentabilität zu beurteilen. Das in der Schweiz übliche System REKOLE $^{\circledR}$ zur betrieblichen Rechnungsstellung an Spitälern ist dazu nicht in der Lage. Ein MIS muss flexibel fixe und variable Kosten unterscheiden und Kosten in Funktion des Umsatzes berechnen können. Zusätzlich muss der Wille vorhanden sein, zugunsten eines gesunden Finanzhaushaltes auf absehbar kostspielige Prestigeprojekte wie den Zusammenschluss von grossen Spitälern (z.B. Inselspital und Spitalnetzwerk Bern) zu verzichten [3].

Es ist offensichtlich, dass sich jeglicher Verbesserungsansatz als politisch dornenvoll erweisen wird.

Leistungsallokation: Sinnlose Investitionen führen zu unnötigen Kapazitätserweiterungen, welche ausgelastet werden wollen. Ein zu grosses Spital kann nur dann rentabel funktionieren, wenn es überflüssige Behandlungen durchführt (Stichwort Qualität der Indikationsstellung) oder wenn es der Konkurrenz Patienten abjagt. In einer Leistungsallokation müsste vom Kanton festgelegt werden: Wer macht was? Bis zu welchem Schwierigkeitsgrad? Mit welcher Qualität?

Die grundlegenden strukturellen «Schwächen» unseres Gesundheitswesens in den angesprochenen Bereichen betreffen alle Kantone gleichermassen. Es ist offensichtlich, dass sich jeglicher Verbesserungsansatz als politisch dornenvoll erweisen wird und eine Realisation allenfalls als ein langwieriger Prozess. Wir haben deswegen diesen Beitrag auch als Utopie bezeichnet. Mit einer klar definierten Strategie könnten allerdings die Bereiche MIS und Leistungsallokation angegangen werden. Es sind dies unmittelbar umsetzbare Massnahmen, welche es den Kantonen erlauben würden, im Gesundheitswesen kostengünstiger zu operieren. In unterschiedlichem Umfang werden diese Mittel von einzelnen Kantonen bereits jetzt erfolgreich eingesetzt. Als wesentlichen weiteren korrigierbaren Mangel sehen wir das Fehlen einer politischen Lobby für den Patienten und Steuerzahler. Solange diejenigen, welche das Gesundheitswesen letztlich finanzieren müssen, keine Stimme haben, wird der lukrative «Markt» Gesundheitswesen ungebremst weiter wachsen. 


\section{Litératur}

1 Schweizerische Akademie der Wissenschaften, 2012, Effizienz, Nutzung und Finanzierung des Gesundheitswesens http://www.akademien-schweiz.ch/index/Schwerpunktthemen/ Gesundheitssystem-im-Wandel/Nachhaltiges-Gesundheitssystem.html

2 OECD Health spending 2014 https://data.oecd.org/healthres/health-spending.htm

3 Hoppeler et al. Mythos Zentralisierung im Spitalwesen. In: Schweiz Ärztezeitung 2014;95(39):1364-6.

4 Hoppeler et al. Kosten und Nutzen kleiner Spitäler. In: Schweiz Ärztezeitung 2012;93(43):1580-3.
5 Bieri, Köchli, 2013, OBSAN Dossier 25; Regionale Unterschiede be der Belastung durch die obligatorischen Gesundheitsausgaben. http://www.gdk-cds.ch/fileadmin/docs/public/gdk/themen/ tarife/praemien/bt_belastung_haushalteobsan_dossier_25.pdf

6 G20/OECD Principles of corporate governance Chapter I, C; «The division of responsibilities among different authorities should be clearly articulated and designed to serve the public interest» https://www.oecd.org/daf/ca/Corporate-Governance-PrinciplesENG.pdf

7 Schlup J, 2016, NZZ vom 30.8.2016, den ambulanten Bereich entwickeln

http://www.nzz.ch/meinung/kommentare/gesundheitskostenden-ambulanten-bereich-entwickeln-ld.113642 